\title{
New constraints and prospects for sub-GeV dark matter scattering off electrons in xenon
}

\author{
Rouven Essig, ${ }^{1, *}$ Tomer Volansky, ${ }^{2, \dagger}$ and Tien-Tien $\mathrm{Yu}^{1,3, \dagger}$ \\ ${ }^{1}$ C.N. Yang Institute for Theoretical Physics, Stony Brook University, Stony Brook, New York 11794, USA \\ ${ }^{2}$ Raymond and Beverly Sackler School of Physics and Astronomy, \\ Tel-Aviv University, Tel-Aviv 69978, Israel \\ ${ }^{3}$ Theoretical Physics Department, CERN, CH-1211 Geneva 23, Switzerland \\ (Received 14 March 2017; revised manuscript received 18 June 2017; published 30 August 2017)
}

\begin{abstract}
We study in detail sub-GeV dark matter scattering off electrons in xenon, including the expected electron recoil spectra and annual modulation spectra. We derive improved constraints using low-energy XENON10 and XENON100 ionization-only data. For XENON10, in addition to including electron-recoil data corresponding to about 1-3 electrons, we include for the first time events corresponding to about 4-7 electrons. Assuming the scattering is momentum independent $\left(F_{\mathrm{DM}}=1\right)$, this strengthens a previous crosssection bound by almost an order of magnitude for dark matter masses above $50 \mathrm{MeV}$. The available XENON100 data corresponds to events with about 4-50 electrons, and leads to a constraint that is comparable to the XENON10 bound above $50 \mathrm{MeV}$ for $F_{\mathrm{DM}}=1$. We demonstrate that a search for an annual modulation signal in upcoming xenon experiments (XENON1T, XENONnT, LZ) could substantially improve the above bounds even in the presence of large backgrounds. We also emphasize that in simple benchmark models of sub-GeV dark matter, the dark matter-electron scattering rate can be as high as one event every ten (two) seconds in the XENON1T (XENONnT or LZ) experiments, without being in conflict with any other known experimental bounds. While there are several sources of backgrounds that can produce single- or few-electron events, a large event rate can be consistent with a dark matter signal and should not be simply written off as purely a detector curiosity. This fact motivates a detailed analysis of the ionization-data ("S2") data, taking into account the expected annual modulation spectrum of the signal rate, as well as the DM-induced electron-recoil spectra, which are another powerful discriminant between signal and background.
\end{abstract}

DOI: $10.1103 /$ PhysRevD.96.043017

\section{INTRODUCTION}

Direct-detection experiments play a crucial role in our quest to identify the nature of dark matter (DM), and the last few years have seen intense interest and significant progress in expanding their sensitivity to particles below $\sim 1 \mathrm{GeV}$. The traditional direct detection techniqueobserving nuclear recoils from DM scattering elastically off nuclei-rapidly loses sensitivity in existing experiments for DM masses below $\sim 1 \mathrm{GeV}$, calling for different approaches. A demonstrated technique with significant potential for improvement is to search for DM scattering off electrons [1]. Various target materials have been investigated, including noble liquids $[1,2]$, semiconductors [1,3-5], scintillators [6], two-dimensional targets [7], and superconductors $[8,9]$. These materials are also sensitive to

\footnotetext{
*rouven.essig@stonybrook.edu

†tomerv@post.tau.ac.il

tien-tien.yu@cern.ch
}

Published by the American Physical Society under the terms of the Creative Commons Attribution 4.0 International license. Further distribution of this work must maintain attribution to the author(s) and the published article's title, journal citation, and DOI. the absorption of ultralight $\mathrm{DM}(\ll \mathrm{MeV})$ by electrons [10-13]. For other direct-detection ideas see [1,14-20]. Direct-detection techniques and complementary probes are summarized in [21].

Currently, the most stringent direct-detection constraint on DM as low as a few MeV comes from XENON10, a two-phase xenon time projection chamber (TPC). When a DM particle scatters off an electron and ionizes a xenon atom in the liquid target, the recoiling electron can ionize other atoms if it has sufficient energy. Electric fields accelerate the ionized electrons through the liquid, across a liquid-gas interface, and propagate electrons through the xenon gas region in which interactions between the electrons and xenon atoms create a scintillation ("S2") signal that is proportional to the number of extracted electrons and detected by photomultiplier tubes. XENON10 [22] has taken data consisting of events that have an S2 signal corresponding to one or more electrons, without an observable prompt scintillation signal ("S1"). The data corresponding to events with three electrons or less $\left(n_{e} \lesssim 3\right)$ were analyzed in [2] and shown to constrain $\mathrm{DM}$ as low as a few MeV.

The main factor limiting the sensitivity of XENON10 is the large number of observed S2-only events and the absence of a background model (to set a constraint, all 
events are conservatively assumed to originate from DM). Plausible origins of these events include the photodissociation of negatively charged impurities; field emission from the cathode; and, especially, electrons that are initially created by highly ionizing background events, but then become trapped in the liquid-gas interface and spontaneously released at a later time [2,22-24]. However, more study is needed to understand and characterize these events. There are several challenges related to the background, including but not limited to multiple origins, time variation, and absence of calibration sources.

In this letter, we derive new constraints from XENON10, including events with $n_{e} \gtrsim 4$. The rate of observed events in this region is lower than for $n_{e} \lesssim 3$, leading to significantly improved constraints for DM masses $m_{\chi} \gtrsim 50 \mathrm{MeV}$. We also analyze S2-only data from XENON100, containing $n_{e} \gtrsim 4$ [25]. We derive the expected recoil spectra for the event rate and the annual and daily modulation amplitude, and show the expected event rates and the implications for a few benchmark DM models.

Other experiments, using semiconductor targets such as germanium $(\mathrm{Ge})$ and silicon $(\mathrm{Si})$, currently have a higher electron-recoil energy threshold and are thus less sensitive by several orders of magnitude than XENON10/100 [5]. Dramatic improvements in sensitivity in the near future are likely with SuperCDMS [5,26], SENSEI [27], and possibly other experiments. Nevertheless, these experiments will initially have target masses of only $\mathcal{O}(1 \mathrm{~kg})$, far less than current and future xenon experiments (Table I). Understanding the S2-only events in two-phase TPCs could thus lead to dramatic improvements in cross-section sensitivity and, as we will show, probe simple and predictive benchmark models. The large exposures will also allow for an annual modulation analysis [28], which can significantly improve upon the current limit even if the background rates are high.

\section{THEORETICAL RATES AND RECOIL SPECTRA}

To calculate the DM-electron scattering rate in liquid xenon, we follow the procedure in [2] (see the Appendix for

TABLE I. Analyzed (XENON10, XENON100, LUX) and approximate projected (XENON1T, LZ, XENONnT) exposures and fiducial masses. We stress that future sensitivities depend on yet-unknown detector-dependent properties, such as the electron lifetime.

\begin{tabular}{lcc}
\hline \hline & Exposure [kg-yrs] & Fiducial mass [kg] \\
\hline XENON10 [22] & 0.041 & 1.2 \\
XENON100 [25] & 29.8 & 48.3 \\
LUX [29] & 119 & 145 \\
XENON1T [30] & 2,000 & 1,000 \\
LZ, XENONnT [30,31] & 15,000 & 5,600 \\
\hline \hline
\end{tabular}

more details). We treat the target electrons as single-particle states of an isolated atom, described by numerical RHF bound wave functions from [32,33]. The velocity-averaged differential ionization cross section for electrons in the $(n, l)$ shell is

$\frac{d\left\langle\sigma_{i o n}^{n l}\right\rangle}{d \ln E_{e r}}=\frac{\bar{\sigma}_{e}}{8 \mu_{\chi e}^{2}} \int q d q\left|f_{i o n}^{n l}\left(k^{\prime}, q\right)\right|^{2}\left|F_{\mathrm{DM}}(q)\right|^{2} \eta\left(v_{\min }\right)$,

where $\eta\left(v_{\min }\right)=\left\langle\frac{1}{v} \theta\left(v-v_{\min }\right)\right\rangle$ is the inverse mean speed for a given velocity distribution as a function of the minimum velocity, $v_{\min }$, required for scattering. We assume a standard Maxwell-Boltzmann velocity distribution with circular velocity $v_{0}=220 \mathrm{~km} / \mathrm{s}$ and a hard cutoff of $v_{\text {esc }}=$ $544 \mathrm{~km} / \mathrm{s}[34,35] . \bar{\sigma}_{e}$ is the DM-free electron scattering cross section at fixed momentum transfer $q=\alpha m_{e}$, while the $q$-dependence of the matrix element is encoded in the DM form-factor $F_{\mathrm{DM}}(q) .\left|f_{\text {ion }}^{n l}\left(k^{\prime}, q\right)\right|^{2}$ is the ionization form factor of an electron in the $(n, l)$ shell with final momentum $k^{\prime}=\sqrt{2 m_{e} E_{e r}}$. We calculate this form factor using the given bound wave functions and unbound wave functions that are obtained by solving the Schrödinger equation with a potential that reproduces the bound wave functions. We consider electrons in the following shells (listed with binding energies in $\mathrm{eV}$ ): $5 p^{6}$ (12.4), $5 s^{2}$ (25.7), $4 d^{10}$ (75.6), $4 p^{6}$ (163.5), and $4 s^{2}$ (213.8). The differential ionization rate is

$$
\frac{d R_{i o n}}{d \ln E_{e r}}=N_{T} \frac{\rho_{\chi}}{m_{\chi}} \sum_{n l} \frac{d\left\langle\sigma_{i o n}^{n l} v\right\rangle}{d \ln E_{e r}},
$$

where $N_{T}$ is the number of target atoms and $\rho_{\chi}=$ $0.4 \mathrm{GeV} / \mathrm{cm}^{3}$ is the local DM density.

We follow [2] to model the conversion from $E_{\mathrm{er}}$ to electron yield, $n_{e}$. The recoiling electron will ionize and excite other atoms, producing $n^{(1)}=\operatorname{Floor}\left(E_{\mathrm{er}} / W\right)$ additional "primary quanta," either observable electrons or (unobservable) scintillation photons. For fiducial values, we choose the probability for the initial electron to recombine with an ion to be $f_{R}=0, W=13.8 \mathrm{eV}$, and the fraction of primary quanta observed as electrons to be $f_{e}=0.83$. To capture the uncertainty in the fiducial values, we vary these parameters in the range $0<f_{R}<0.2,12.4<$ $W<16 \mathrm{eV}$, and $0.62<f_{e}<0.91$. The ranges for the parameters was chosen considering the available data and literature [36-39]. In addition to primary quanta, if DM ionizes an inner-shell electron, $n^{(2)}=\operatorname{Floor}\left(\left(E_{i}-E_{j}\right) / W\right)$ secondary quanta can be created by photons produced in the subsequent outer-to-inner-shell electron transitions with binding energies $E_{i, j}$. The number of secondary electrons produced follows a binomial distribution with $n^{(1)}+n^{(2)}$ trials and success probability $f_{e}$.

In Fig. 1, we show the recoil spectra as a function of $n_{e}$ for a hypothetical xenon detector with $1000 \mathrm{~kg}$-years of exposure for $F_{\mathrm{DM}}=1$ (top) and $F_{\mathrm{DM}}=\alpha^{2} m_{e}^{2} / q^{2}$ (bottom). 


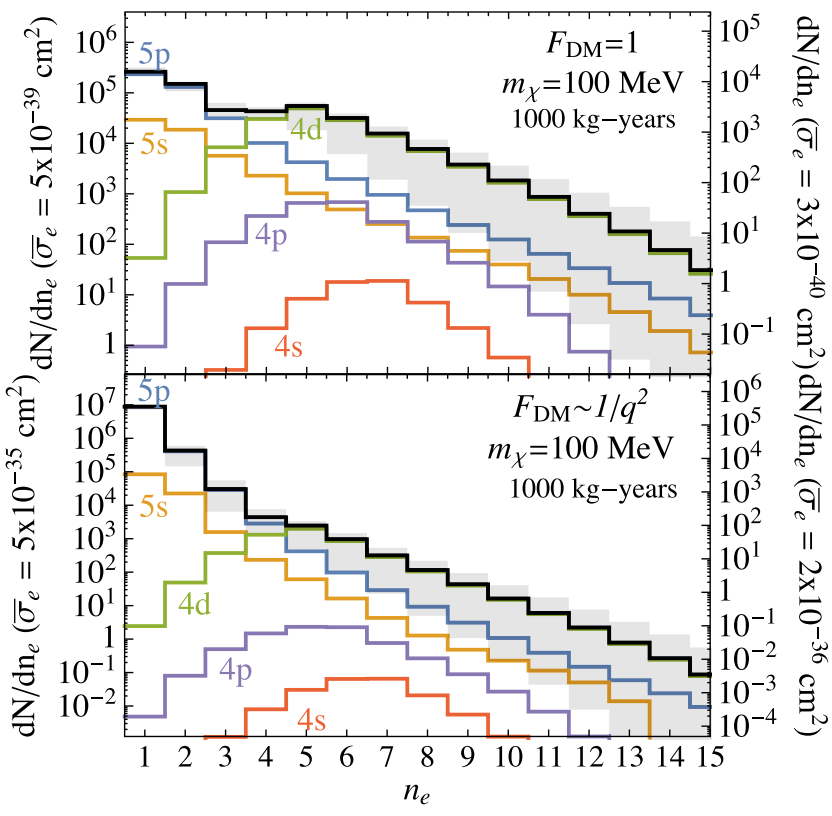

FIG. 1. Top (bottom): Spectrum of expected number of events for DM-electron scattering in xenon, for $m_{\chi}=100 \mathrm{MeV}$ and $1000 \mathrm{~kg}$-years for $F_{\mathrm{DM}}=1\left(\alpha^{2} m_{e}^{2} / q^{2}\right)$. For the left axes, we set $\bar{\sigma}_{e}$ to the maximum allowed values by current constraints for two popular benchmark models; for the right axes, the indicated $\bar{\sigma}_{e}$ produces the correct relic abundance. Colored lines show individual contributions from various xenon electron shells, while the gray band encompasses the spectrum when varying the secondary ionization model. See text for details.

The colored lines show individual contributions from different shells, while the black line shows their sum (for fiducial values). Gray bands show the variation away from the fiducial values discussed above.

To emphasize the importance of studying electron recoil events at current and upcoming xenon experiments, we have fixed $\bar{\sigma}_{e}$ to specific values that are allowed by simple and predictive benchmark models [1,5,40-46] and further below. We consider the DM (a Dirac fermion or complex scalar $\chi$ ) to be charged under a broken $U(1)_{D}$ gauge force, mediated by a kinetically-mixed dark photon, $A^{\prime}$, with mass $m_{A^{\prime}}$. The $A^{\prime}$ mediates DM-electron scattering, and $F_{\mathrm{DM}}(q)=1\left(\alpha^{2} m_{e}^{2} / q^{2}\right)$ for a heavy (ultralight) dark photon. The left axis for top (bottom) plot of Fig. 1 shows the event rate for $\bar{\sigma}_{e}$ fixed to the maximum value allowed by current constraints for $m_{A^{\prime}}=3 m_{\chi}\left(m_{A^{\prime}} \ll \mathrm{keV}\right)$, while the right axis of the top (bottom) plot fixes $\bar{\sigma}_{e}$ so that scalar (fermion) DM obtains the correct relic abundance from thermal freeze-out (freeze-in). Clearly, a large number of DM events could be seen in upcoming detectors. These results are easily rescaled to other DM models that predict DM-electron scattering.

\section{NEW XENON10 AND XENON100 BOUNDS}

We now recalculate the bounds from XENON10 data [2] (15 kg-days), including for the first time events with $n_{e} \gtrsim 4$, as well as from XENON100 data [25] (30 kg-years). Since the experimental observable is the number of photoelectrons (PE) produced by an event, we convert $n_{e}$ to PE. An event with $n_{e}$ electrons produces a gaussian distributed number of PE with mean $n_{e} \mu$ and width $\sqrt{n_{e}} \sigma$, where $\mu=27(19.7)$ and $\sigma=6.7(6.2)$ for XENON10 (XENON100). We multiply the signal with the trigger and acceptance efficiencies from $[2,25,36]$, where the acceptance is taken to be a flat 0.92 , and then bin both the signal and data in steps of $27 \mathrm{PE}$ (20 PE), starting from $14 \mathrm{PE}$ (80 PE) for XENON10 (XENON100). The first bin for the XENON100 analysis is 80-90 PE, corresponding to a width of 0.5 electron. We require that the resulting signal is less than the data at $90 \%$ C.L. in each bin. For XENON10, the 90\% C.L. upper bounds on the rates (after unfolding the efficiencies) are $r_{1}<15.18$, $r_{2}<3.37, r_{3}<0.95, r_{4}<0.35, r_{5}<0.35, r_{6}<0.15 r_{7}<$ 0.35 counts kg ${ }^{-1}$ day $^{-1}$, corresponding to bins $b_{1}=[14,41]$, $b_{2}=[41,68] \ldots, b_{7}=[176-203] \mathrm{PE}$; for XENON100, we find $r_{4}<0.17, \quad r_{5}<0.24, \quad r_{6}<0.17$ counts kg ${ }^{-1}$ day $^{-1}$
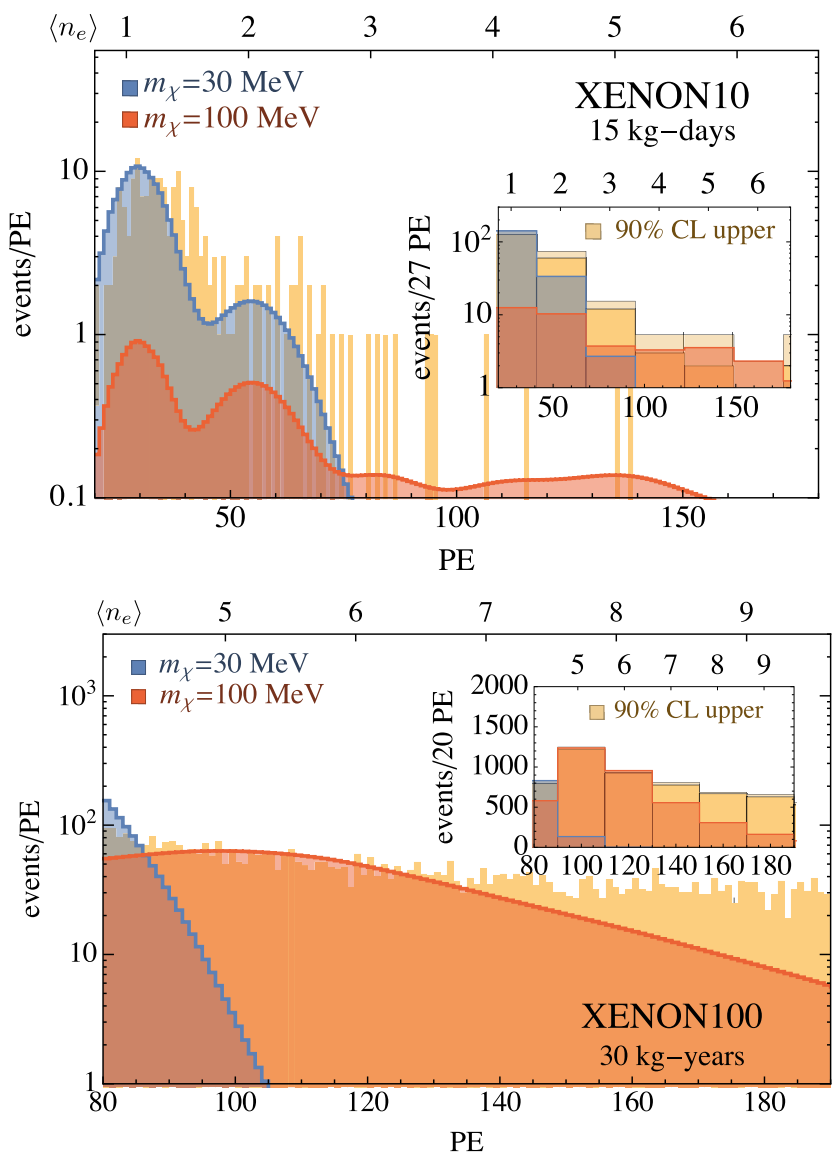

FIG. 2. Observed number of events versus photoelectrons (PE) in XENON10 (top) [22] and XENON100 (bottom) [25] (tan). DM spectra are shown for $m_{\chi}=30 \mathrm{MeV}$ (blue) and $100 \mathrm{MeV}$ (red) with a cross section fixed at our derived 90\% C.L. limit (we assume fiducial values for the secondary ionization model). Insets show spectra in bins of $27 \mathrm{PE}$ (20 PE), the mean number of PE created by one electron in XENON10 (XENON100). 


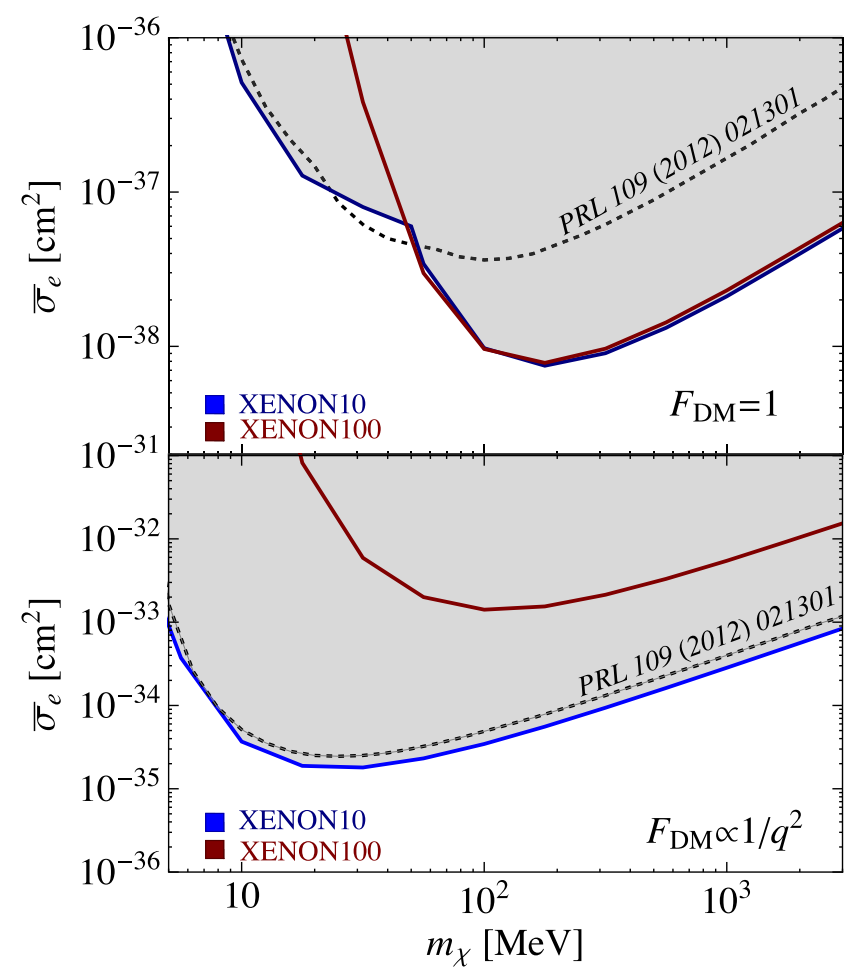

FIG. 3. $90 \%$ C.L. limit on the DM-electron scattering cross section from XENON10 data (blue) and XENON100 data (red) for $F_{\mathrm{DM}}=1$ (top) \& $F_{\mathrm{DM}}=\alpha^{2} m_{e}^{2} / q^{2}$ (bottom). Dotted black lines show XENON10 bounds from [2].

corresponding to bins $b_{4}=[80,90], b_{5}=[90,110], b_{6}=$ $[110,130]$ PE.

Figure 2 shows the two data sets in PE and two sample DM spectra. Figure 3 shows the strongest XENON10 and XENON100 limit combined across all bins, and a comparison with the XENON10 bound derived in [2]. In the Appendix, we show cross-section bounds for the individual PE bins, taking into account the systematic uncertainties from the secondary ionization model. For $F_{\mathrm{DM}}=1$, the inclusion of the high-PE bins in XENON10 significantly improves upon the bound from [2] for $m_{\chi} \gtrsim 50 \mathrm{MeV}$ (small differences at lower masses are from the limitsetting procedure). The new XENON10 and XENON100 bounds are comparable for $m_{\chi} \gtrsim 50 \mathrm{MeV}$. For $F_{\mathrm{DM}}=$ $\alpha^{2} m_{e}^{2} / q^{2}$, the low PE bins determine the bound, and XENON100 is therefore not competitive due to its high analysis threshold.

\section{MODULATION}

A useful discriminant between signal and background is the annual modulation of the signal rate [28] due to the Sun's motion through the DM halo. Figure 4 shows $f_{\text {mod }}$ versus $n_{e}$, where $f_{\text {mod }}=\frac{R_{\max }-R_{\min }}{2 R_{\text {avg }}}$ is the modulation amplitude, derived by calculating the rates for the average Earth velocity and varying it by $\pm 15.0 \mathrm{~km} / \mathrm{s}$. The $f_{\text {mod }}$ spectrum

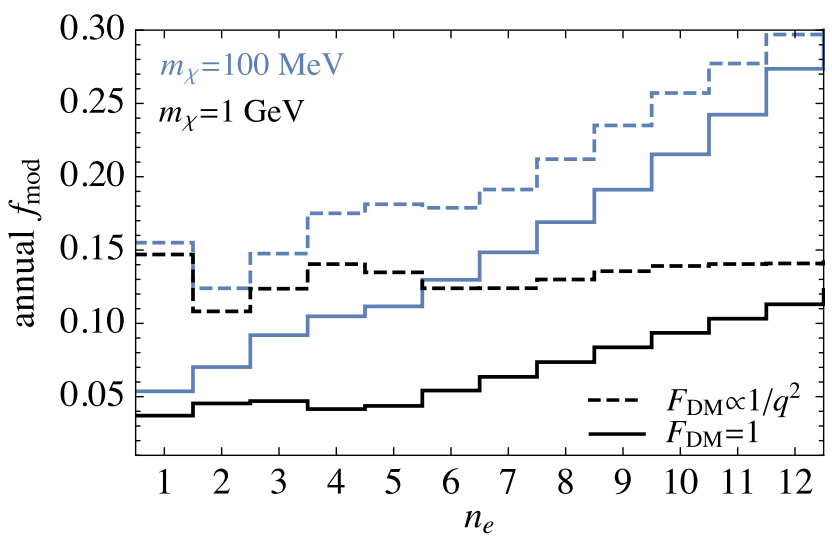

FIG. 4. Annual modulation amplitude for $F_{\mathrm{DM}}=1$ (solid) \& $F_{\mathrm{DM}}=\alpha^{2} m_{e}^{2} / q^{2} \quad$ (dashed) for $m_{\chi}=100 \mathrm{MeV}$ (blue) \& $1 \mathrm{GeV}$ (black).

is distinctive, which should provide a helpful discriminant between signal and background. The significance of a signal $S$ over a flat background $B$ is then given by sig $=\frac{f_{\bmod } S}{\sqrt{S+B}}$.

To demonstrate the power of an annual modulation search, we imagine that a future detector with $1000 \mathrm{~kg}$-years of exposure observes the same S2-only event rate and spectrum as observed in XENON10 data, $R_{\mathrm{Xe} 10}$, as given in the previous section. Requiring the signal rate to be less than the observed event rate yields the same constraints as with XENON10 data, $\bar{\sigma}_{e, \mathrm{Xe} 10}$. However, an annual modulation analysis would potentially see a signal of high statistical significance, and in the absence of one a fraction of the observed event rate must be background. Requiring the significance of the annual modulation signal to be less than sig, the expected sensitivity is

$$
\bar{\sigma}_{e}^{\bmod }=\frac{\operatorname{sig} \times \bar{\sigma}_{e, \mathrm{Xe} 10}}{f_{\bmod } \sqrt{R_{\mathrm{Xe} 10} \times \text { exposure }}} .
$$

We calculate $\bar{\sigma}_{e}^{\text {mod }}$ for bins of $n_{e}=0.5-1.5,1.5-2.5, \ldots$ and show with a blue line the best sensitivity across all bins in Fig. 5 for $s i g=1.65(90 \%$ CL) (see the Appendix for sensitivities from each bin). Similarly, a red solid line shows $\bar{\sigma}_{e}^{\text {mod }}$ assuming the future observed rates/spectrum correspond to the current XENON100 rate/spectrum. We overlay these lines on the DM benchmark models discussed above. However, largescale liquid xenon detectors may not be able to detect single to few electrons in the entirety of the target mass, and so the usable fiducial volume will be smaller than the quoted values. Nevertheless, while hypothetical, this analysis emphasizes the power of an annual modulation analysis. Deriving a more realistic limit would require a dedicated study of possible time-dependent backgrounds. 

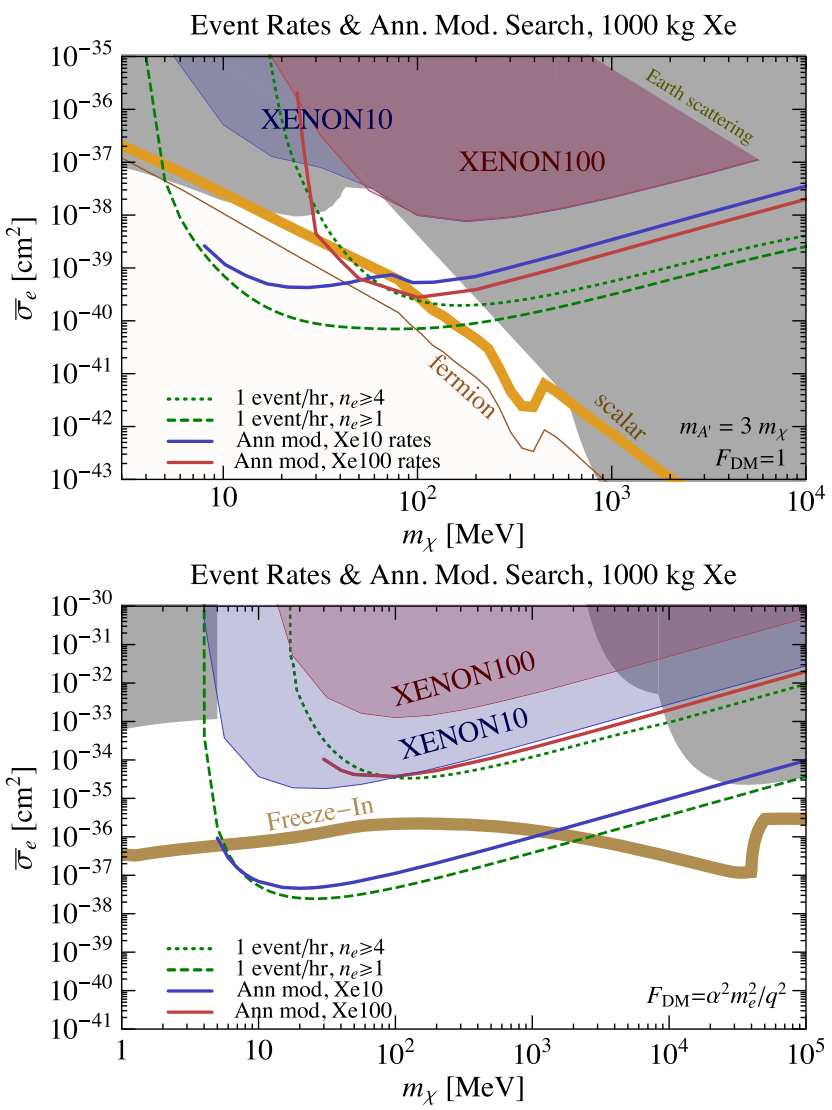

FIG. 5. Sensitivity reach from an annual modulation analysis with a hypothetical $1000 \mathrm{~kg}$ detector and 1-year exposure, assuming the observed spectrum and data rate are the same as in XENON10 [22] (solid blue) or XENON100 [25] (solid red). DM-electron scattering event rates assuming a 1-electron (4-electron) threshold are shown in dashed (dotted) green. Blue (red) shaded regions show our XENON10 (XENON100) limits. These lines/regions are overlaid on several simple and predictive benchmark models for $\mathrm{DM}(\chi)$ scattering off electrons via a dark photon $A^{\prime}$. Top: $\left(F_{\mathrm{DM}}=1\right)$ A complex scalar obtains the correct relic density from thermal freeze-out (light orange), while a fermion, which obtains its correct relic abundance from an initial asymmetry, must have $\bar{\sigma}_{e}$ above the dark brown line (assuming no additional annihilation channels) to avoid indirect-detection constraints [47-49]. Bottom: $\left(F_{\mathrm{DM}}=\alpha^{2} m_{e}^{2} / q^{2}\right)$ Fermion DM coupled to an ultralight mediator $A^{\prime}$ obtains the correct relic density from freeze-in (thick brown line). Gray regions show constraints as in [5], updated on the top plot with data from MiniBooNE [50] and BABAR [51]. Due to earthscattering effects [52], no XENON10/100 limit exists in the top right region.

\section{LARGE EVENT RATES}

To further emphasize the importance of understanding the electron recoil events in xenon TPCs, we show the expected event rates in Fig. 5 for a $1000 \mathrm{~kg}$ detector for two thresholds, $n_{e} \geq 1$ and $n_{e} \geq 4$. We see that the benchmark DM models predict large event rates. For example, Dirac fermion DM coupled to the $A^{\prime}$ that obtains its abundance from an initial asymmetry could produce about one event every two seconds at LZ. This underscores the point that while there are several sources of backgrounds that can produce single- or few-electron events, a large event rate can be consistent with a DM signal and should not be simply written off as a detector curiosity.

\section{CONCLUSIONS}

We derived new constraints on DM-electron scattering, improving upon the previous bound, and showed spectra for the expected number of electrons and the modulation amplitude. While there are several possible detectorspecific origins of the observed XENON10/100 events, in principle almost all the observed events could originate from DM-electron scattering without coming into conflict with other existing DM constraints. This is not the case when interpreting these events as arising from few-GeV DM recoiling elastically off nuclei $[22,25]$, which is excluded by existing results from e.g. LUX [53] and CDMSlite [54]. Moreover, simple and predictive DM benchmark models predict large event rates in current and future xenon TPCs. An expanded and dedicated effort by the xenon collaborations to understand the origin of their low-energy electron recoil data is thus imperative and well worth the effort.

\section{ACKNOWLEDGMENTS}

We would like to thank especially Aaron Manalaysay and Peter Sorensen for many insightful discussions. We also thank Ran Budnik, Daniel McKinsey, Matt Pyle, and Jingke $\mathrm{Xu}$ for useful discussions. We are also very grateful to Jeremy Mardon for contributions at the beginning of this project as well as many useful discussions. R.E. is supported by the Department of Energy Early Career research program DESC0008061 and through a Sloan Foundation Research Fellowship. T.-T. Y. is also supported by Grant No. DESC0008061. T. V. is supported by the European Research Council (ERC) under the EU Horizon 2020 Programme (ERC-CoG-2015-Proposal No. 682676 LDMThExp), by the PAZI foundation, by the GermanIsraeli Foundation (Grant No. I-1283- 303.7/2014) and by the I-CORE Program of the Planning Budgeting Committee and the Israel Science Foundation (Grant No. 1937/12). For its hospitality, T.-T. Y. thanks the Aspen Center for Physics, where part of this work was completed and which is supported by National Science Foundation Grant No. PHY-1066293.

\section{APPENDIX}

Here we provide additional details to the calculations described in the main text. We also show spectra plots for additional DM masses, as well as the XENON10/ XENON100 limits and the prospects for an annual modulation analysis from each PE bin. For completeness, we also 
TABLE II. Xenon shells and energies. "Photon energy" refers to energy of deexcitation photons for outer-shell electrons deexciting to lower shells. This photon can subsequently photoionize, creating additional quanta. The range of additional quanta takes into account that the higher energy shell may have more than one available lower energy shell to deexcite into. For our limits, we take the minimum of this range.

\begin{tabular}{lccccc}
\hline \hline Shell & $5 p^{6}$ & $5 s^{2}$ & $4 d^{10}$ & $4 p^{6}$ & $4 s^{2}$ \\
\hline Binding energy [eV] & 12.4 & 25.7 & 75.6 & 163.5 & 213.8 \\
Photon energy [eV] & - & 13.3 & 63.2 & 87.9 & 201.4 \\
Additional quanta & 0 & 0 & 4 & $6-10$ & $3-15$ \\
\hline \hline
\end{tabular}

show the expected daily modulation of the signal rate due to the Earth's rotation.

\section{Theoretical rates}

We first quote additional formulas that are required for the rate calculation (see also [2,5]). The velocity-averaged differential ionization cross section for electrons in the $(n, l)$ shell is given in Eq. (1). The full expression for $v_{\min }$ is

$$
v_{\min }=\frac{\left(\left|E_{\text {binding }}^{n l}\right|+E_{\mathrm{er}}\right)}{q}+\frac{q}{2 m_{\chi}},
$$

where $E_{\text {binding }}^{n l}$ is the binding energy of the shell and $q$ is the momentum transfer from the DM to the electron. The form factor for ionization of an electron in the $(n, l)$ shell with final momentum $k^{\prime}=\sqrt{2 m_{e} E_{\mathrm{er}}}$ is given by

$$
\begin{aligned}
& \left|f_{\text {ion }}^{n l}\left(k^{\prime}, q\right)\right|^{2} \\
& =\frac{4 k^{\prime 3}}{(2 \pi)^{3}} \sum_{l^{\prime} L}(2 l+1)\left(2 l^{\prime}+1\right)(2 L+1) \\
& \quad \times\left[\begin{array}{lll}
l & l^{\prime} & L \\
0 & 0 & 0
\end{array}\right]^{2}\left|\int r^{2} d r R_{k^{\prime} l^{\prime}}(r) R_{n l}(r) j_{L}(q r)\right|^{2},
\end{aligned}
$$

where $[\cdots]$ is the Wigner $3-j$ symbol and $j_{L}$ are the spherical Bessel functions. We solve for the radial wavefunctions $R_{k^{\prime} l^{\prime}}(r)$ of the outgoing unbound electrons taking the radial Schrödinger equation with a central potential $Z_{\text {eff }}(r) / r$. This central potential is determined from the initial electron wavefunction by assuming that it is a bound state of the same potential. We include the shells listed in Table II.

\section{Electron and photoelectron yields}

We provide additional details to convert the recoiling electron's recoil energy into a specific number of electrons. The relevant quantities are

$$
\begin{gathered}
E_{\mathrm{er}}=\left(n_{\gamma}+n_{e}\right) W, \\
n_{\gamma}=N_{\mathrm{ex}}+f_{R} N_{i}, \\
n_{e}=\left(1-f_{R}\right) N_{i} .
\end{gathered}
$$
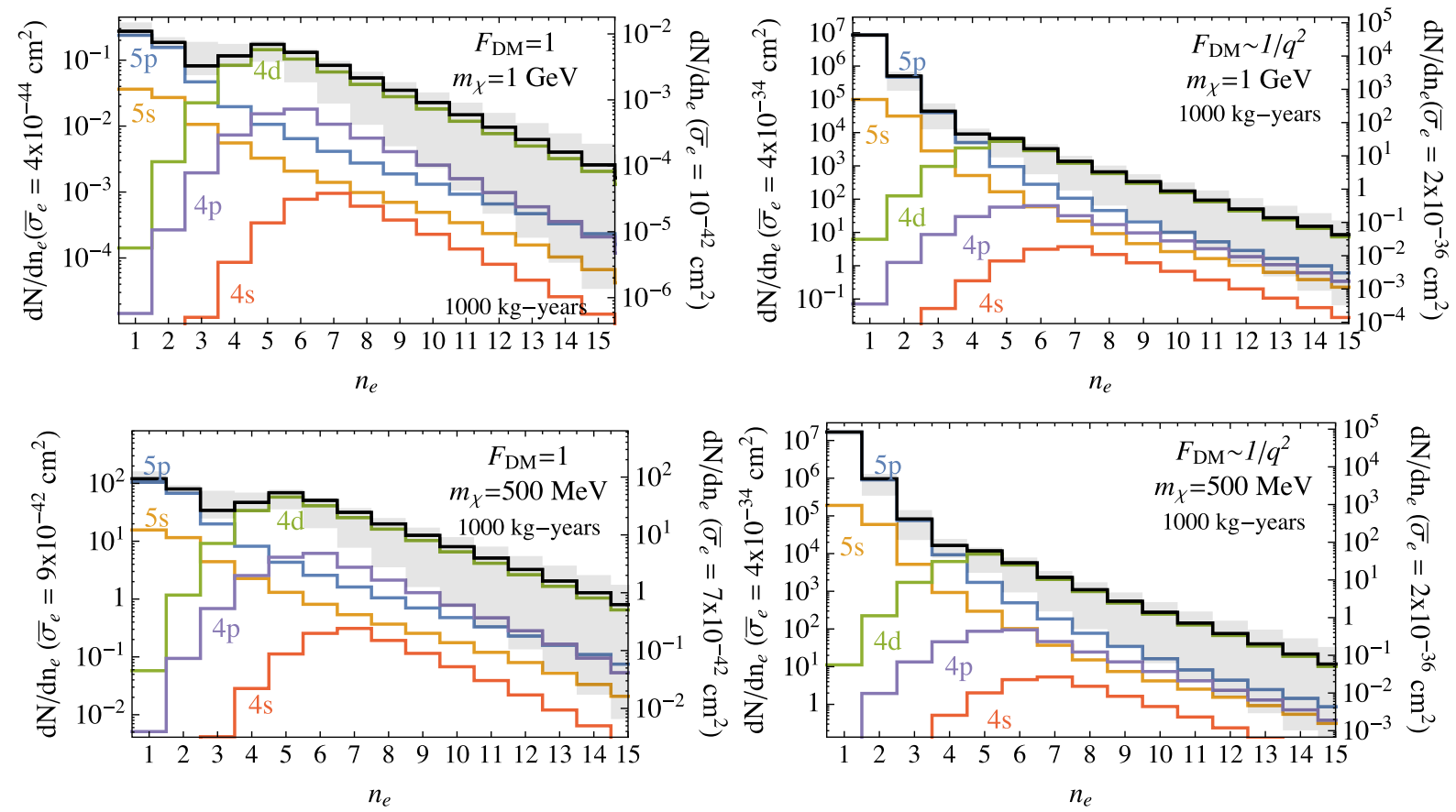

FIG. 6. Expected number of events as a function of number of electrons observed for $1000 \mathrm{~kg}$ yr of xenon. The left axis sets $\bar{\sigma}_{e}$ to the maximum allowed value by current constraints while the right axis sets $\bar{\sigma}_{e}$ to the predicted value for a freeze-out (freeze-in) model for $F_{\mathrm{DM}}=1\left(\alpha^{2} m_{e}^{2} / q^{2}\right)$, respectively. The different colored lines show the contributions from the various xenon shells while the gray band encodes the uncertainties associated with the secondary ionization processes. 
$E_{\text {er }}$ is the amount of deposited energy from the primary electron, which results in a number of observable electrons, $n_{e}$, unobservable scintillation photons, $n_{\gamma}$, and heat. $W$ is the energy needed to produce a single quanta (photon or electron). We take $W=13.8 \pm 0.9 \mathrm{eV}$ as the average energy [37]. $E_{\text {er }}$ can create both a number of ions, $N_{i}$, and a number of excited atoms $N_{\mathrm{ex}}$, where $N_{\mathrm{ex}} / N_{i} \simeq 0.2$ at energies above a $\mathrm{keV}[37,38]$. We take into account the possibility that the primary electron and secondary ions can recombine, which is described by a modified ThomasImel recombination model [55], and assume that the fraction of ions that can recombine, $f_{R}$, is effectively zero at low energy. This implies that $n_{e}=N_{i}$ and $n_{\gamma}=N_{\mathrm{ex}}$. The fraction of initial quanta observed as electrons is given by $f_{e}=\left(1-f_{R}\right) /\left(1+N_{\mathrm{ex}} / N_{i}\right) \simeq 0.83$ [56]. To capture the uncertainty in $f_{R}, W$, and $N_{\mathrm{ex}} / N_{i}$, we calculate the rates and limits varying these parameters over the ranges $0<f_{R}<0.2,0.1<N_{\mathrm{ex}} / N_{i}<0.3$, and $12.4<$ $W<16 \mathrm{eV}$. For our fiducial values, we set $f_{e}=0.83$, $f_{R}=0, W=13.8 \mathrm{eV}$.
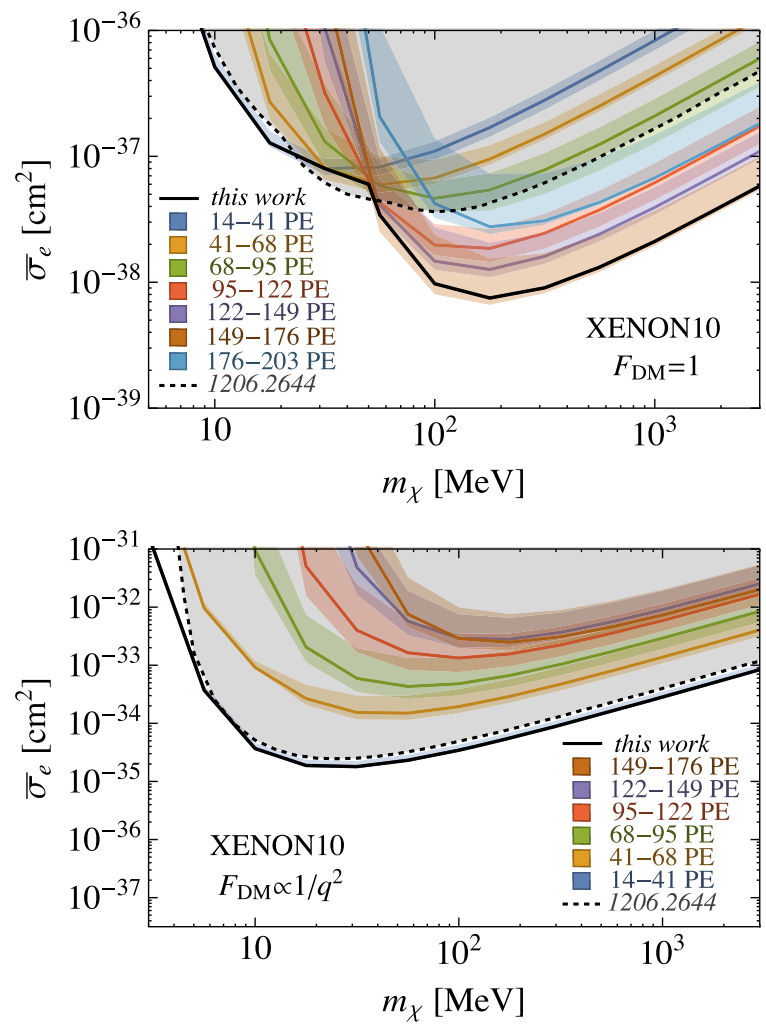

FIG. 7. New XENON10 limit (black) obtained as described in the text. The colored bands are from the uncertainty in the secondary ionization model. The shaded gray region shows the parameter space previously excluded by the 1,2 , and 3 electron XENON10 data. By including the contributions to the S2 signal from 14PE to 203PE, we see that the limits improve considerably for DM masses above $\sim 50 \mathrm{MeV}$ for $F_{\mathrm{DM}}=1$, while there is no improvement due to the momentum suppression for $F_{\mathrm{DM}}=\alpha^{2} m_{e}^{2} / q^{2}$.
For each primary electron with energy $E_{\text {er }}$, we assume that there are additional $n^{(1)}=\operatorname{Floor}\left(E_{\mathrm{er}} / W\right)$ quanta created. Furthermore, we assume that the photons associated with the deexcitation of the next-to-outer shells $(5 s, 4 d, 4 p, 4 s)$, which have energies $(13.3,63.2,87.9$, 201.4) $\mathrm{eV}$, can photoionize to create an additional $n^{(2)}=$ $\left(n_{5 s}, n_{4 d}, n_{4 p}, n_{4 s}\right)=(0,4,6-10,3-15)$ quanta, respectively (see Table II). The range in values for the $4 p$ and $4 s$ shells takes into consideration that there may be more than one outer-shell electron available that can deexcite down to them. For example, if the $4 d$ shell deexcites to $4 p, 6$ additional quanta are created, while if the $5 s$ shell deexcites to $4 p$, it would create 10 additional quanta. For our fiducial values, we take the lower number of quanta to be conservative. However, the choice of the number of additional quanta only affects $n_{e}>6$, and even here the difference in event rate is smaller than the uncertainties due to the modeling of the secondary ionization.

The total number of electrons is given by $n_{e}=n_{e}^{\prime}+n_{e}^{\prime \prime}$, where $n_{e}^{\prime}$ is the primary electron and $n_{e}^{\prime \prime}$ are the secondary electrons produced. $n_{e}^{\prime}=0$ or 1 with probability $f_{R}$ or
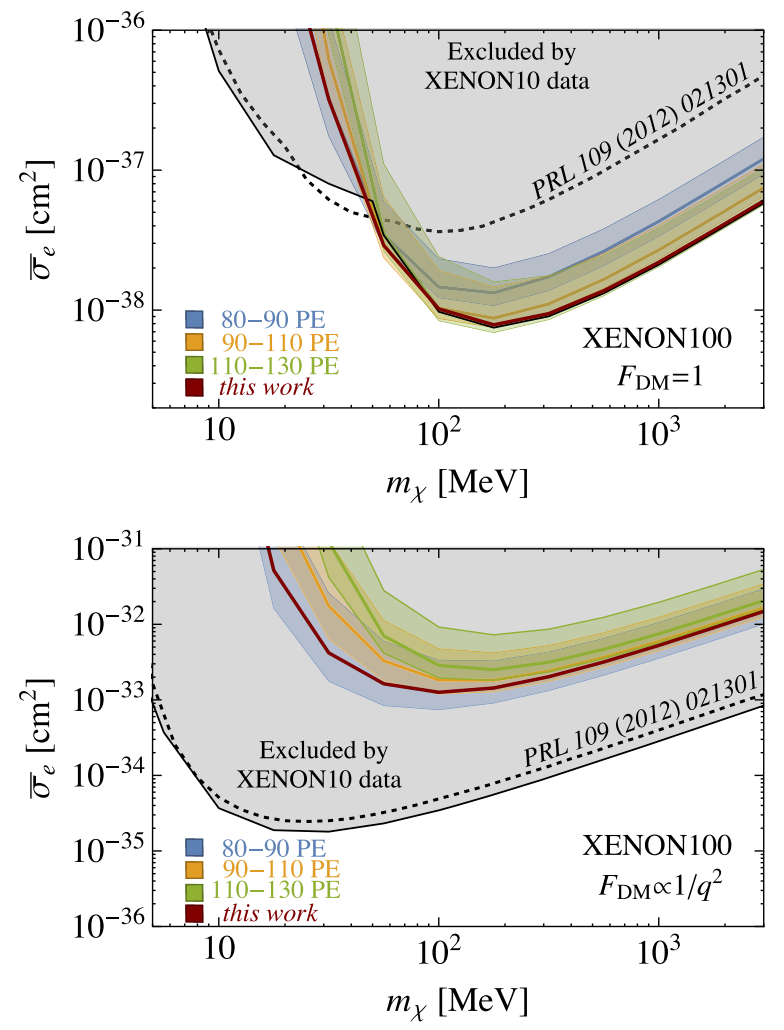

FIG. 8. New limit obtained using the XENON100 data (red). The XENON100 data starts at $80 \mathrm{PE}$ electrons, so we show the individual limits for the 80-90, 90-110, and 110-130 PE bins. The colored bands are from the uncertainty in the secondary ionization model. The shaded gray region shows the parameter space excluded by our updated XENON10 analysis, while the dotted black line shows the XENON10 bound from [2]. 
Annual Modulation, 90\%CL

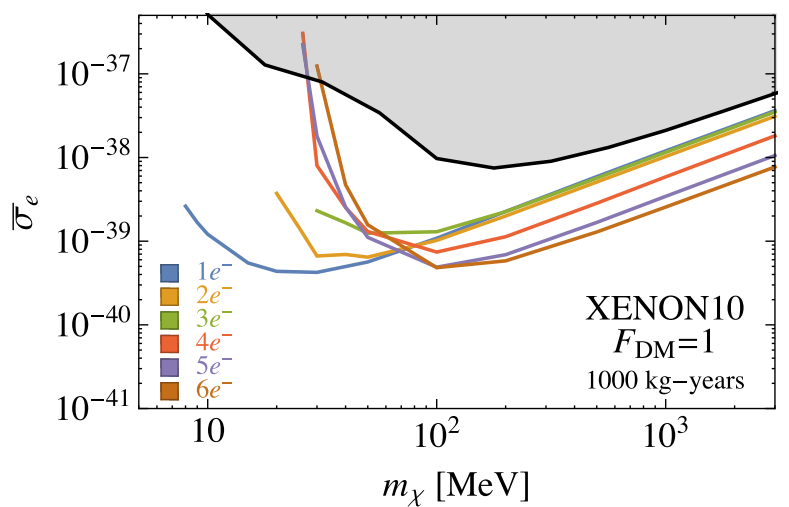

Annual Modulation, 90\%CL

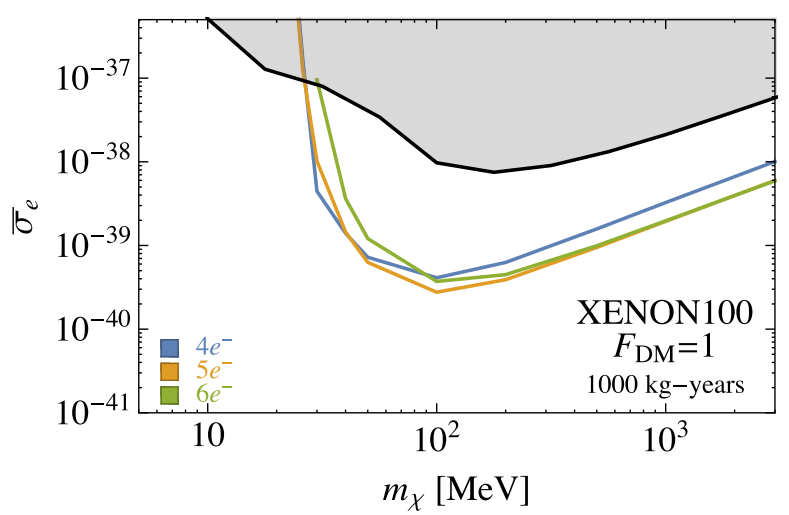

Annual Modulation, $90 \% \mathrm{CL}$

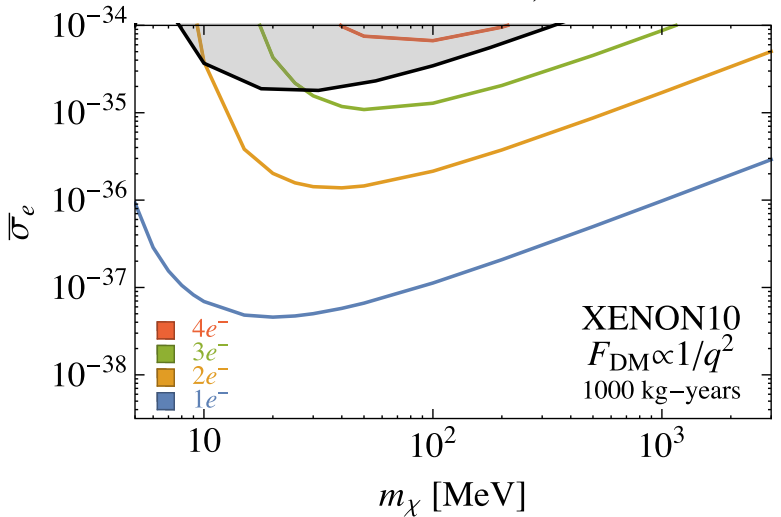

Annual Modulation, 90\%CL

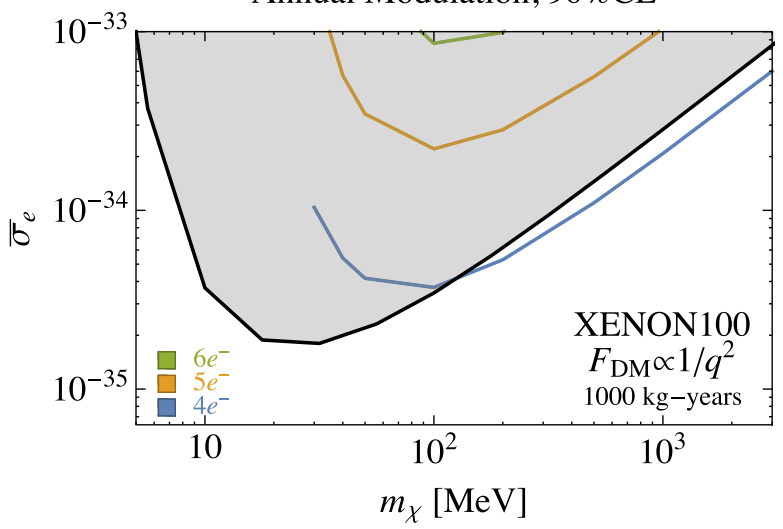

FIG. 9. Individual bin sensitivities to the $90 \%$ C.L. annual modulation reach for a $1000 \mathrm{~kg}$ yr xenon detector. The background rates and spectra are taken to be the XENON10 (XENON100) rates scaled up to $1000 \mathrm{~kg}$ yr for the top (bottom) panels (see also text and Fig. 5).

$1-f_{R}$, respectively, while $n_{e}^{\prime \prime}$ follows a binomial distribution with $n^{(1)}+n^{(2)}$ trials and success probability $f_{e}$.

Given this conversion from $E_{\mathrm{er}}$ into $n_{e}$, we can calculate the differential rate as a function of number of electrons. In addition to the $m_{\chi}=100 \mathrm{MeV}$ spectra shown in the main text, we show the spectra for $m_{\chi}=500 \mathrm{MeV}$ and $1 \mathrm{GeV}$ in Fig. 6.

\section{XENON10 and XENON100 constraints for individual photoelectron bins}

In the main text, we show the cross-section limits from the XENON10 and XENON100 data using the fiducial values above. In Figs. 7 and 8, we show the individual limits for each PE bin as well as the uncertainty bands due to the secondary ionization model.

\section{Modulation}

In Fig. 5, we showed how an annual modulation analysis of a hypothetical xenon detector with an exposure of $1000 \mathrm{~kg}$ yr could significantly improve on current constraints even if the background rates are significant. In Fig. 5, we only showed the best constraints across all individual $n_{e}$ bins. In Fig. 9, we show the individual $n_{e}$ bins. Furthermore, for completeness, we also show the daily modulation amplitude due to the Earth's rotation with respect to the DM wind. The daily modulation is calculated by modifying the average Earth velocity by $\pm 0.23 \mathrm{~km} / \mathrm{s}$ to obtain the maximum and minimum rates. We show the daily modulation fraction in Fig. 10, where we see that the daily modulation fraction is about an order of magnitude smaller than that of the annual modulation.

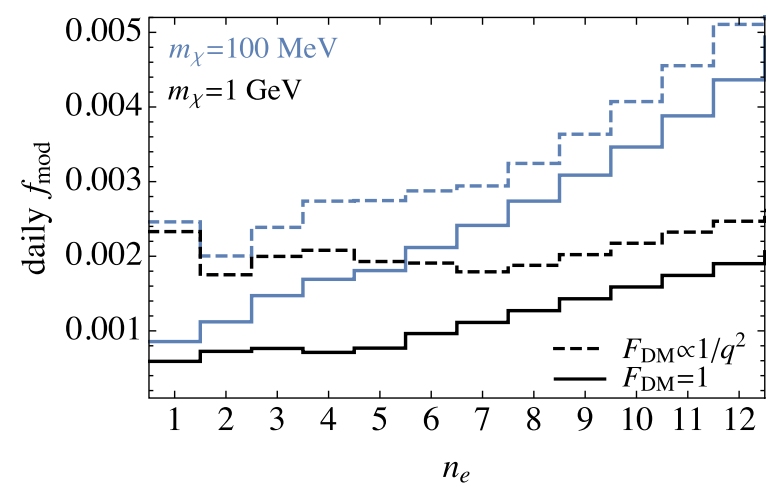

FIG. 10. Daily modulation amplitude for $F_{\mathrm{DM}}=1$ (solid) and $F_{\mathrm{DM}}=\alpha^{2} m_{e}^{2} / q^{2}$ (dashed) for $m_{\chi}=100 \mathrm{MeV}$ (blue) and $1 \mathrm{GeV}$ (black). 
[1] R. Essig, J. Mardon, and T. Volansky, Phys. Rev. D 85, 076007 (2012).

[2] R. Essig, A. Manalaysay, J. Mardon, P. Sorensen, and T. Volansky, Phys. Rev. Lett. 109, 021301 (2012).

[3] P. W. Graham, D. E. Kaplan, S. Rajendran, and M. T. Walters, Phys. Dark Universe 1, 32 (2012).

[4] S. K. Lee, M. Lisanti, S. Mishra-Sharma, and B. R. Safdi, Phys. Rev. D 92, 083517 (2015).

[5] R. Essig, M. Fernandez-Serra, J. Mardon, A. Soto, T. Volansky, and T.-T. Yu, J. High Energy Phys. 05 (2016) 046.

[6] S. Derenzo, R. Essig, A. Massari, A. Soto, and T.-T. Yu, Phys. Rev. D 96, 016026 (2017).

[7] Y. Hochberg, Y. Kahn, M. Lisanti, C. G. Tully, and K. M. Zurek, Phys. Lett. B 772, 239 (2017).

[8] Y. Hochberg, M. Pyle, Y. Zhao, and K. M. Zurek, J. High Energy Phys. 08 (2016) 057.

[9] Y. Hochberg, Y. Zhao, and K. M. Zurek, Phys. Rev. Lett. 116, 011301 (2016).

[10] H. An, M. Pospelov, and J. Pradler, Phys. Rev. Lett. 111, 041302 (2013).

[11] H. An, M. Pospelov, J. Pradler, and A. Ritz, Phys. Lett. B 747, 331 (2015).

[12] I. M. Bloch, R. Essig, K. Tobioka, T. Volansky, and T.-T. Yu, J. High Energy Phys. 06 (2017) 1.

[13] Y. Hochberg, T. Lin, and K. M. Zurek, Phys. Rev. D 95, 023013 (2017).

[14] R. Essig, J. Mardon, O. Slone, and T. Volansky, Phys. Rev. D 95, 056011 (2017).

[15] R. Budnik, O. Cheshnovsky, O. Slone, and T. Volansky (to be published).

[16] K. Schutz and K. M. Zurek, Phys. Rev. Lett. 117, 121302 (2016).

[17] S. Knapen, T. Lin, and K. M. Zurek, Phys. Rev. D 95, 056019 (2017).

[18] C. Kouvaris and J. Pradler, Phys. Rev. Lett. 118, 031803 (2017).

[19] C. McCabe, arXiv:1702.04730.

[20] P. C. Bunting, G. Gratta, T. Melia, and S. Rajendran, Phys. Rev. D 95, 095001 (2017).

[21] R. Essig, J. A. Jaros, W. Wester, P. H. Adrian, S. Andreas et al., arXiv:1311.0029.

[22] J. Angle et al. (XENON10 Collaboration), Phys. Rev. Lett. 107, 051301 (2011); 110, 249901(E) (2013).

[23] E. Aprile et al. (XENON100 Collaboration), J. Phys. G 41, 035201 (2014).

[24] P. Sorensen, arXiv:1702.04805.

[25] E. Aprile et al. (XENON Collaboration), Phys. Rev. D 94, 092001 (2016); 95, 059901 (2017).

[26] P. Cushman et al., arXiv:1310.8327.

[27] J. Tiffenberg (SENSEI Collaboration), SENSEI project, in Proceedings of 3rd Berkeley Workshop on the Direct Detection of Dark Matter 2016, https://indico.physics.lbl .gov/indico/event/311/contribution/28/material/slides/1.pdf.
[28] A. K. Drukier, K. Freese, and D. N. Spergel, Phys. Rev. D 33, 3495 (1986).

[29] D. S. Akerib et al. (LUX Collaboration), Phys. Rev. Lett. 118, 021303 (2017).

[30] E. Aprile et al. (XENON Collaboration), J. Cosmol. Astropart. Phys. 04 (2016) 027.

[31] D. S. Akerib et al. (LZ Collaboration), arXiv:1509.02910.

[32] C. Bunge, J. Barrientos, and A. Bunge, At. Data Nucl. Data Tables 53, 113 (1993).

[33] Experimental results may suggest that liquid xenon has a band structure with a gap of about $9.2 \mathrm{eV}$ [34]. In this case, our calculation, which assumes an ionization energy of $12.4 \mathrm{eV}$, may underestimate slightly the true scattering rate.

[34] M. C. Smith et al., Mon. Not. R. Astron. Soc. 379, 755 (2007).

[35] W. Dehnen and J. Binney, Mon. Not. R. Astron. Soc. 298, 387 (1998).

[36] E. Aprile et al. (XENON Collaboration), Astropart. Phys. 34, 679 (2011).

[37] T. Doke, A. Hitachi, J. Kikuchi, K. Masuda, H. Okada, and E. Shibamura, Jpn. J. Appl. Phys. 41, 1538 (2002).

[38] E. Aprile, K. L. Giboni, P. Majewski, K. Ni, and M. Yamashita, Phys. Rev. B 76, 014115 (2007).

[39] C. Dahl, Ph.D. thesis, Princeton University, 2009.

[40] C. Boehm and P. Fayet, Nucl. Phys. B683, 219 (2004).

[41] N. Borodatchenkova, D. Choudhury, and M. Drees, Phys. Rev. Lett. 96, 141802 (2006).

[42] B. Batell, M. Pospelov, and A. Ritz, Phys. Rev. D 80, 095024 (2009).

[43] X. Chu, T. Hambye, and M. H. Tytgat, J. Cosmol. Astropart. Phys. 05 (2012) 034.

[44] T. Lin, H.-B. Yu, and K. M. Zurek, Phys. Rev. D 85, 063503 (2012).

[45] E. Izaguirre, G. Krnjaic, P. Schuster, and N. Toro, Phys. Rev. Lett. 115, 251301 (2015).

[46] J. Alexander et al., arXiv:1608.08632.

[47] M. S. Madhavacheril, N. Sehgal, and T. R. Slatyer, Phys. Rev. D 89, 103508 (2014).

[48] P. A. R. Ade et al. (Planck Collaboration), Astron. Astrophys. 594, A13 (2016).

[49] R. Essig, E. Kuflik, S. D. McDermott, T. Volansky, and K. M. Zurek, J. High Energy Phys. 11 (2013) 193.

[50] A. A. Aguilar-Arevalo et al. (MiniBooNE Collaboration), Phys. Rev. Lett. 118, 221803 (2017).

[51] J. P. Lees et al. (BABAR Collaboration), arXiv:1702.03327.

[52] T. Emken, C. Kouvaris, and I. M. Shoemaker, Phys. Rev. D 96, 015018 (2017).

[53] D. S. Akerib et al. (LUX Collaboration), Phys. Rev. Lett. 116, 161302 (2016).

[54] J. Thomas and D. A. Imel, Phys. Rev. A 36, 614 (1987).

[55] R. Agnese et al. (SuperCDMS Collaboration), Phys. Rev. Lett. 116, 071301 (2016).

[56] P. Sorensen and C.E. Dahl, Phys. Rev. D 83, 063501 (2011). 